\title{
Alloantigen-specific regulatory $T$ cells generated with a chimeric antigen receptor
}

\author{
Katherine G. MacDonald, ${ }^{1}$ Romy E. Hoeppli, ${ }^{1}$ Qing Huang, ${ }^{2}$ Jana Gillies, ${ }^{1}$ Dan S. Luciani, ${ }^{1}$ Paul C. Orban, ${ }^{1}$ \\ Raewyn Broady, ${ }^{2}$ and Megan K. Levings ${ }^{1}$ \\ 'Department of Surgery and 'Department of Medicine, University of British Columbia, and Child and Family Research Institute, Vancouver, British Columbia, Canada.
}

\begin{abstract}
Adoptive immunotherapy with regulatory $T$ cells (Tregs) is a promising treatment for allograft rejection and graft-versushost disease (GVHD). Emerging data indicate that, compared with polyclonal Tregs, disease-relevant antigen-specific Tregs may have numerous advantages, such as a need for fewer cells and reduced risk of nonspecific immune suppression. Current methods to generate alloantigen-specific Tregs rely on expansion with allogeneic antigen-presenting cells, which requires access to donor and recipient cells and multiple MHC mismatches. The successful use of chimeric antigen receptors (CARs) for the generation of antigen-specific effector $T$ cells suggests that a similar approach could be used to generate alloantigenspecific Tregs. Here, we have described the creation of an HLA-A2-specific CAR (A2-CAR) and its application in the generation of alloantigen-specific human Tregs. In vitro, A2-CAR-expressing Tregs maintained their expected phenotype and suppressive function before, during, and after A2-CAR-mediated stimulation. In mouse models, human A2-CAR-expressing Tregs were superior to Tregs expressing an irrelevant CAR at preventing xenogeneic CVHD caused by HLA-A2+ $\mathrm{T}$ cells. Together, our results demonstrate that use of CAR technology to generate potent, functional, and stable alloantigen-specific human Tregs markedly enhances their therapeutic potential in transplantation and sets the stage for using this approach for making antigen-specific Tregs for therapy of multiple diseases.
\end{abstract}

\section{Introduction}

The essential role of regulatory $\mathrm{T}$ cells (Tregs) in preventing autoimmunity and controlling responses to alloantigens is well established. Multiple Treg-based cell therapy approaches are now being tested in the clinic, with early promising results reported in prevention of graft-versus-host disease (GVHD) after allogeneic hematopoietic stem cell transplantation (HSCT) (1-3) and maintenance of $\mathrm{C}$-peptide levels in type 1 diabetes $(4,5)$. The results of these phase I trials indicate that Treg therapy seems to be well tolerated and possibly efficacious, but that there may be a transient risk of generalized immunosuppression (6).

Data from animal studies indicate that the potency and specificity of Treg therapy can be markedly enhanced by the use of antigen-specific cells. For example, in models of autoimmunity, antigen-specific Tregs are superior to polyclonal Tregs in reducing disease: Tregs isolated from pancreatic lymph nodes or pulsed with islet antigen are significantly better at preventing or curing type 1 diabetes than are polyclonal Tregs (7-11), and Tregs expressing an autoantigen-specific transgenic T cell receptor (TCR) are superior to polyclonal Tregs at suppressing central nervous system inflammation in a model of experimental autoimmune encephalomyelitis (EAE) (12). Similarly, alloantigen-specific Tregs, enriched by alloantigen-stimulated expansion in vitro or engineered to express a TCR

\section{Related Commentary: p. 1248}

Authorship note: P.C. Orban and R. Broady contributed equally to this work. Conflict of interest: The authors have declared that no conflict of interest exists. Submitted: May 13, 2015; Accepted: February 4, 2016.

Reference information: J Clin Invest. 2016;126(4):1413-1424. doi:10.1172/JCI82771. transgene, are more effective than polyclonal Tregs at preventing rejection of organ and tissue grafts (13-17). Although limited, there is some evidence that Tregs expanded with alloantigens also effectively prevent GVHD (18) and that in vivo induction of antigen-specific Tregs promotes acceptance of hematopoietic allografts without GVHD (19). Humanized mouse models have shown similar results: alloantigen-expanded human Tregs are more potent suppressors of skin graft rejection than are polyclonal Tregs $(20,21)$.

An alternate approach to overexpressing transgenic TCRs or antigen-stimulated expansion to enrich for antigen-specific $\mathrm{T}$ cells is the use of chimeric antigen receptors (CARs), in which T cells are genetically engineered to express extracellular single-chain $\mathrm{Ab}$ $(\mathrm{scFv})$ antigen-binding domains fused to intracellular signaling domains $(22,23)$. Tregs expressing CARs specific for model antigens have been tested (24-28), leading us to hypothesize that this approach could be used in the context of transplantation. Here, we describe what we believe is a new approach to generating potent alloantigen-specific Tregs, using a CAR targeting HLA-A2.

\section{Results}

Construction and validation of an A2-CAR. We aimed to generate a new CAR specific for HLA-A2, as this is a commonly mismatched antigen in transplantation, with a prevalence of approximately $50 \%$ in those of mixed European descent (29), and HLA-A mismatching is associated with poor outcomes after transplantation (30). As detailed in Methods, we generated lentiviral vectors encoding an HLA-A2-specific CAR by cloning and sequencing the heavy- and light-chain variable regions of the BB7.2 mAb and fusing the resulting $\mathrm{ScFv}$ to portions of $\mathrm{CD} 8, \mathrm{CD} 28$, and $\mathrm{CD} 3 \zeta$ in a second-generation CAR structure (ref. 31 and Figure 1, A and B). A 
A

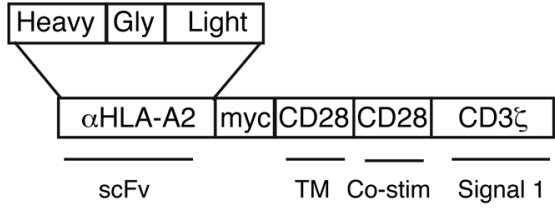

B
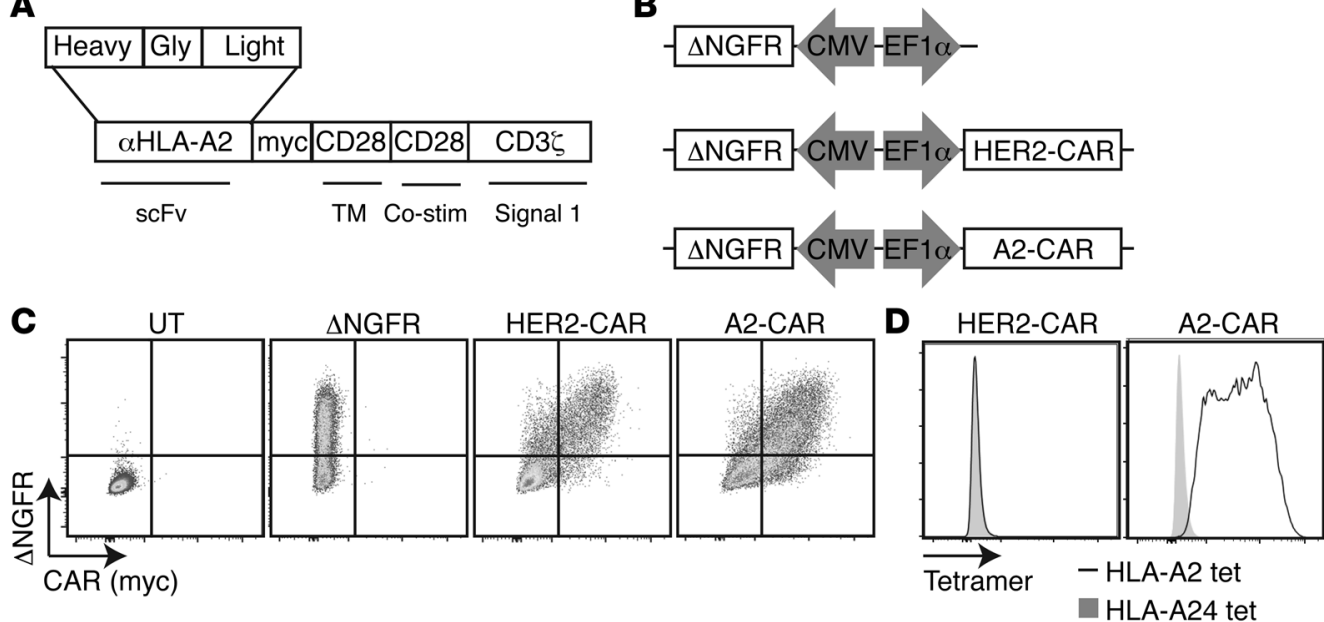

Figure 1. Construction, expression, and antigen specificity of an HLA-A2-specific CAR. (A) Schematic of domains in the A2-CAR. Gly, glycine serine linker; Myc, Myc-tag; TM, transmembrane. (B) Schematic of bidirectional lentiviral vectors encoding the truncated nerve growth factor receptor ( $\triangle$ NGFR) as a selectable marker under a minimal CMV promoter and CARs specific for HLA-A2 or HER2 under the EF1 $\alpha$ promoter. (C) 293T cells were transfected with an empty vector ( $\triangle N$ NGFR), or a vector encoding a HER2-CAR, or the A2-CAR. Surface expression was measured by detection of the Myc-epitope tag by flow cytometry. UT, untransfected. (D) 293T cells expressing the HER2-CAR or A2-CAR were stained with HLA-A2 or HLA-A24 tetramers (tet). Data are representative of 2 independent experiments. it was important to exclude the possibility that high expression of a CAR in Tregs might result in loss of the expected Treg phenotype. A2-CARexpressing Tregs maintained significantly higher expression of FOXP3 than Tconvs and preserved a high degree of demethylation of the Tregspecific demethyled region (TSDR) of the FOXP3 locus (Figure 2, D and E), consistent with other reports of demethylation observed in naive Tregs sorted by flow cytometry (38). High expression of other canonical Treg markers, including CD25, Helios, and CTLA-4, was also preserved in A2-CAR-expressing Tregs (Supplemental Figure 1A; supplemental material available online with this article;

second-generation CAR containing CD28 was chosen because of the well-characterized importance of this costimulatory molecule in Treg development and function (32). A lentivector encoding a well-characterized HER2-specific CAR (HER2-CAR) (33) served as a negative control. Surface expression of the HLA-A2-specific CAR (A2-CAR) was confirmed by transient transfection of 293T cells and flow cytometric staining for the extracellular Myc epitope (Figure 1C). mAbs reformatted to $\mathrm{scFv}$ may have reduced antigen binding or specificity depending on their components $(34,35)$. We therefore used tetramers made from HLA-A2 or HLA-A24 to confirm that the specificity of binding to HLA-A2 was retained. As shown in Figure 1D, cells expressing the A2-CAR bound to HLAA2 tetramers, but not to control HLA-A24 tetramers.

Generation of A2-CAR Tregs. To test the function of the A2-CAR in Tregs, we sorted CD25 ${ }^{\text {hi }}$ CD $45 \mathrm{RA}^{+}$cells from peripheral blood, as this population is known to have homogeneous expression of FOXP3 and to have the highest expansion potential (Figure 2A and ref. 36). $\mathrm{CD} 25^{\mathrm{lo}} \mathrm{CD} 45 \mathrm{RA}^{+}$cells were sorted in parallel as the conventional $\mathrm{T}$ cell (Tconv) control. As outlined in Figure 2B, Tregs (or control Tconvs) were stimulated, transduced, and purified as $\triangle \mathrm{NGFR}^{+}$cells, then expanded for an additional 6 days. At the end of 13 to 14 days of culture, cell-surface expression of A2-CAR or HER2-CAR on both Tregs and Tconvs was confirmed with flow cytometry (Figure 2C). Interestingly, while similar proportions of Treg and Tconv populations were positive for CAR expression, on a per-cell basis, A2-CAR expression appeared to be higher in both cell types than expression of the HER2-CAR. Even when CAR expression intensity was normalized to the expression of $\triangle \mathrm{NGFR}$, A2-CAR-expressing cells showed significantly higher levels of CAR expression than cells expressing HER2-CAR.

The effect of CAR expression on the phenotype and function of human Tregs is unknown, and since high expression of CARs has been linked to undesired antigen-independent CAR activation (37),
doi:10.1172/JCI82771DS1). In comparison with CAR-expressing Tconvs, A2-CAR Tregs also did not contain significant proportions of IL-2- or IFN- $\gamma$-producing cells (Supplemental Figure 1B).

To determine whether A2-CAR Tregs preserved their in vitro suppressive function when activated via their endogenous TCR (i.e., not via the A2-CAR), $\alpha \mathrm{CD} 3 / \mathrm{CD} 28 \mathrm{mAbs}$ were used to stimulate HLA-A2- peripheral blood mononuclear cells (PBMCs) alone or in the presence of decreasing ratios of A2-CAR Tregs. As shown in Supplemental Figure 1C, A2-CAR Tregs suppressed the proliferation of $\mathrm{CD}^{+} \mathrm{T}$ cells in a dose-dependent manner. Finally, cultures of Tregs expressing either CAR had high viability, ranging from $86 \%$ to $96 \%$ (Supplemental Figure 1D). Thus, expression of a second-generation CAR comprising domains from $\mathrm{scFv}, \mathrm{CD} 8 \alpha$, $\mathrm{CD} 28$, and $\mathrm{CD} 3 \zeta$ does not alter the phenotype, stability, or in vitro function of human Tregs. tigated how A2-CAR stimulation compares with traditional TCR activation of Tregs. We first tested the ability of TCR versus CAR activation to stimulate intracellular signaling pathways. A2-CAR Tregs or Tconvs were TCR stimulated with anti-CD3 mAbs or CAR stimulated by crosslinking the extracellular Myc-tag, and relative levels of phospho-ZAP70 were determined by flow cytometry. TCR and CAR stimulation activated ZAP70 equally, with no statistically significant differences between Tregs and Tconvs (Figure 3A).

A2-CAR-expressing Tregs or Tconvs were next left unstimulated or stimulated via the CAR, with K562 cells expressing HLAA2, or via the TCR, with K562 cells loaded with $\alpha \mathrm{CD} 3 / \mathrm{CD} 28$ $\mathrm{mAbs}$, for 24 hours. CAR stimulation resulted in upregulation of the canonical activation marker CD69 in both Tregs and Tconvs to levels that tended to be higher than those of TCR-stimulated cells (Figure 3B). In contrast, in comparison with Tconvs, Tregs had significantly lower expression of CD154 (CD4OL) upon activation
A2-CAR-mediated stimulation activates Tregs. We next inves- 
A

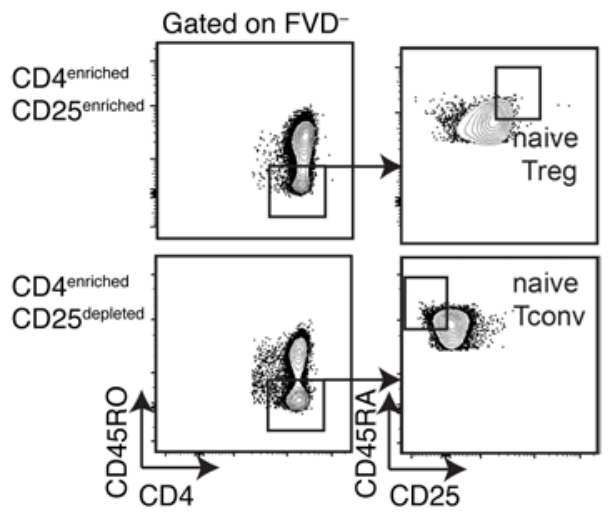

B

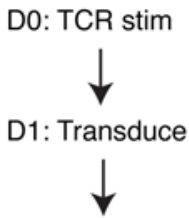

D7: $\triangle$ NGFR purify and TCR stim

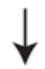

D13-14: $T$ cell assays
C

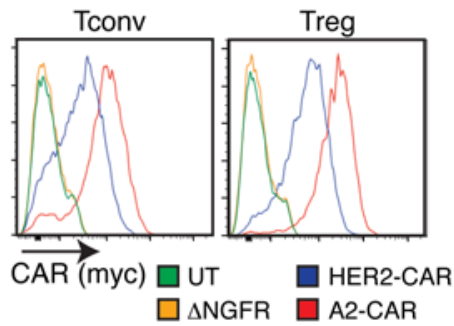

$\% \mathrm{CAR}^{+}$

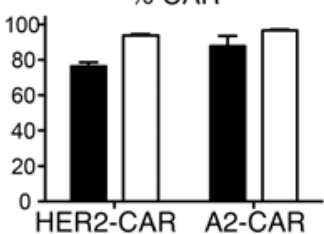

CAR MFI/NGFR MFI

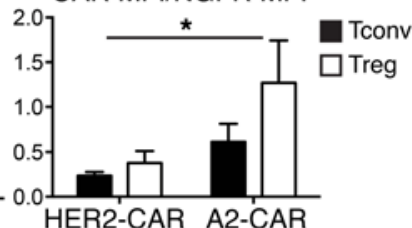

D

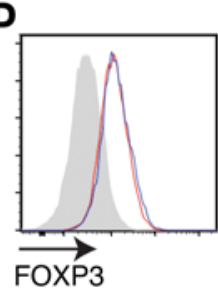

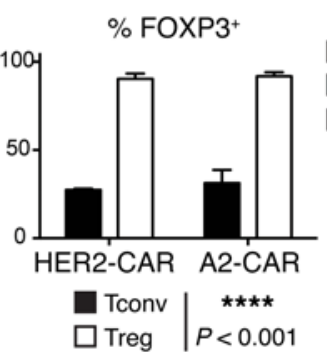
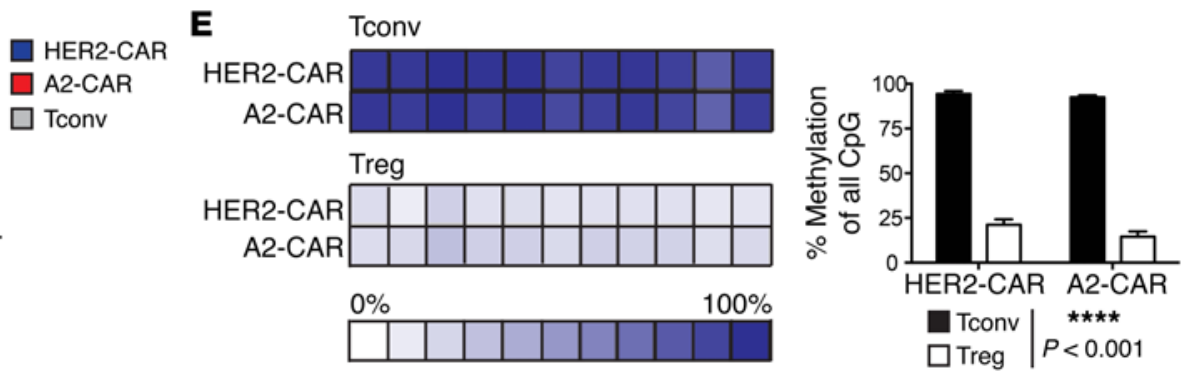

Figure 2. Generation of HLA-A2-CAR Tregs. (A) Sorting strategy to isolate naive Tregs and Tconvs from CD25-enriched and CD25-depleted CD4+ cells, respectively. (B) Schematic diagram outlining the protocol to transduce naive human T cells with lentiviral vectors encoding CARs. (C) Extracellular expression of CARs was assessed by staining for the Myc-epitope tag. The average proportion of CAR ${ }^{+}$cells in $\triangle N G F R$-selected cells is summarized. The average CAR MFI was determined relative to $\triangle$ NGFR MFI on the same cell $(n=3)$. (D) Expression of FOXP3 was assessed at day 14; representative data are on the left and averaged data are on the right $(n=3)$. (E) TSDR methylation was determined by pyrosequencing of cells from males at day $14(n=4)$. Left panel shows data from each CpG residue, with averaged data combing all CpGs to the right. Significance determined by 2-way ANOVA. Data represent mean \pm SEM. ${ }^{*} P<0.05$.

with either TCR or CAR, consistent with the known low CD154 expression in human Tregs (39).

In comparison with TCR-mediated activation, A2-CAR-mediated stimulation of Tregs caused markedly greater upregulation of proteins associated with Treg function. The proportion of CTLA-4-expressing Tregs was significantly higher in CAR-versus TCR-stimulated cultures as was the mean fluorescence intensity (MFI) of expression (Figure 3C). CAR-stimulated Tregs also had significantly higher surface expression of latency-associated peptide (LAP) and glycoprotein A repetitions predominant (GARP), the inactive form of TGF- $\beta$ and one of its receptors, respectively, compared with TCR-stimulated cells (Figure 3D). Importantly, A2-CAR-mediated Treg activation did not alter the cytokine phenotype of Tregs. Whereas A2-CAR-stimulated Tconvs produced significant amounts of IFN- $\gamma$, TNF- $\alpha$, and IL-2 (Figure 3E), A2-CAR-stimulated Tregs did not make significant amounts of any of these cytokines. Thus, short-term (24-48 hours) CAR stimulation does not alter the expected Treg phenotype.

A2-CAR-mediated stimulation and expansion do not alter the Treg phenotype. We next asked how long-term CAR-mediated stimulation of Tregs affects their phenotype and function. To address this question, we analyzed the phenotype of A2-CAR-expressing Tregs or Tconvs (generated as in Figure 2B) stimulated through their endogenous TCR (with K562.64 cells loaded with $\alpha \mathrm{CD} 3 / 28$ mAbs) or with K562 cells expressing HLA-A2 over multiple days.
Both TCR- and CAR-stimulated Tregs maintained the expected expression pattern of Treg lineage markers, with high expression of FOXP3 (both percentage and MFI) and CD25 and low expression of CD127 (Figure 4A).

After 2 weeks, CAR stimulation resulted in a significantly higher fold expansion and increased viability of Tregs compared with TCRexpanded cells (Figure 4B) and retained the Tregs' suppressive activity (Figure 4C). TCR- and CAR-stimulated Tregs remained more than $85 \% \mathrm{FOXP}^{+}$and had similar levels of FOXP3 on a per-cell basis, with percentage of both FOXP3 ${ }^{+}$and MFI significantly higher than in Tconvs (Figure 4D). However, there was a slight downregulation of the A2-CAR MFI in CAR-expanded cells, possibly due to receptor internalization, since the overall proportion of $\mathrm{CAR}^{+}$cells was similar after TCR or CAR expansion. Tregs expanded through their CAR also maintained high expression of CD25 and CTLA-4 (Figure 4E).

A2-CAR Tregs mediate HLA-A2-specific suppression. We then asked whether A2-CAR Tregs preferentially interacted with HLAA2-expressing PBMCs. A2-CAR and HER2-CAR Tregs were cultured with a mixture of HLA-A2 ${ }^{+}$and HLA-A2- PBMCs, which were labeled with a red or green dye, respectively, and the number of interactions between the Tregs and the red versus green PBMCs was observed over time by fluorescence microscopy (Figure 5A). The total number of cells per field was similar, but we found significantly more interactions between A2-CAR Tregs and HLA$\mathrm{A2}^{+}$PBMCs than between A2-CAR Tregs and HLA-A2- PBMCs or 
A A2-CAR Treg
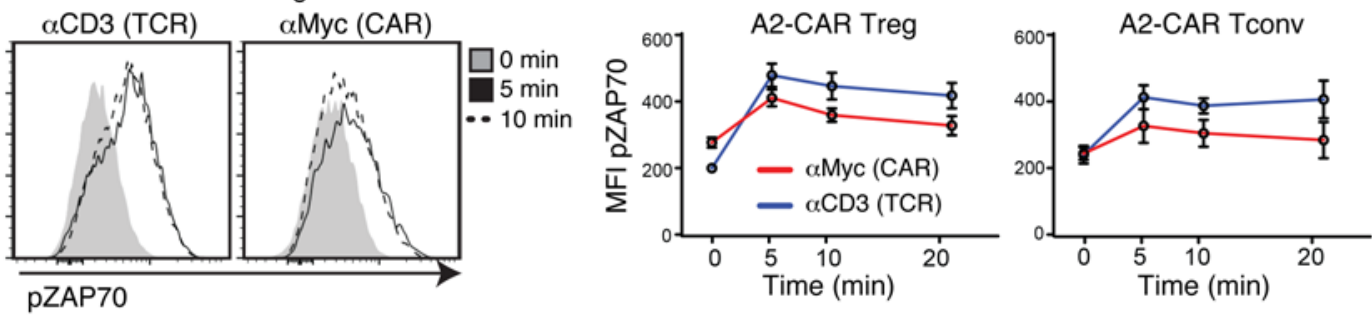

B
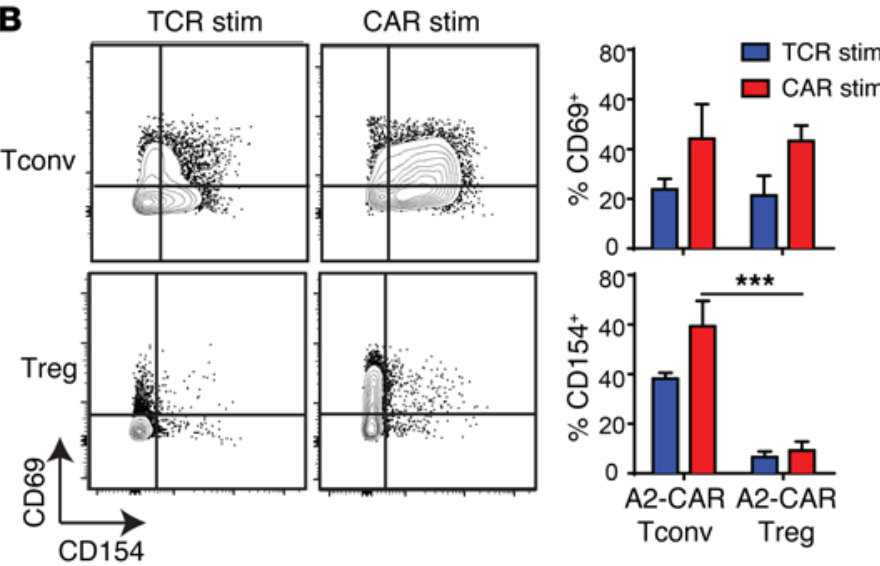

C
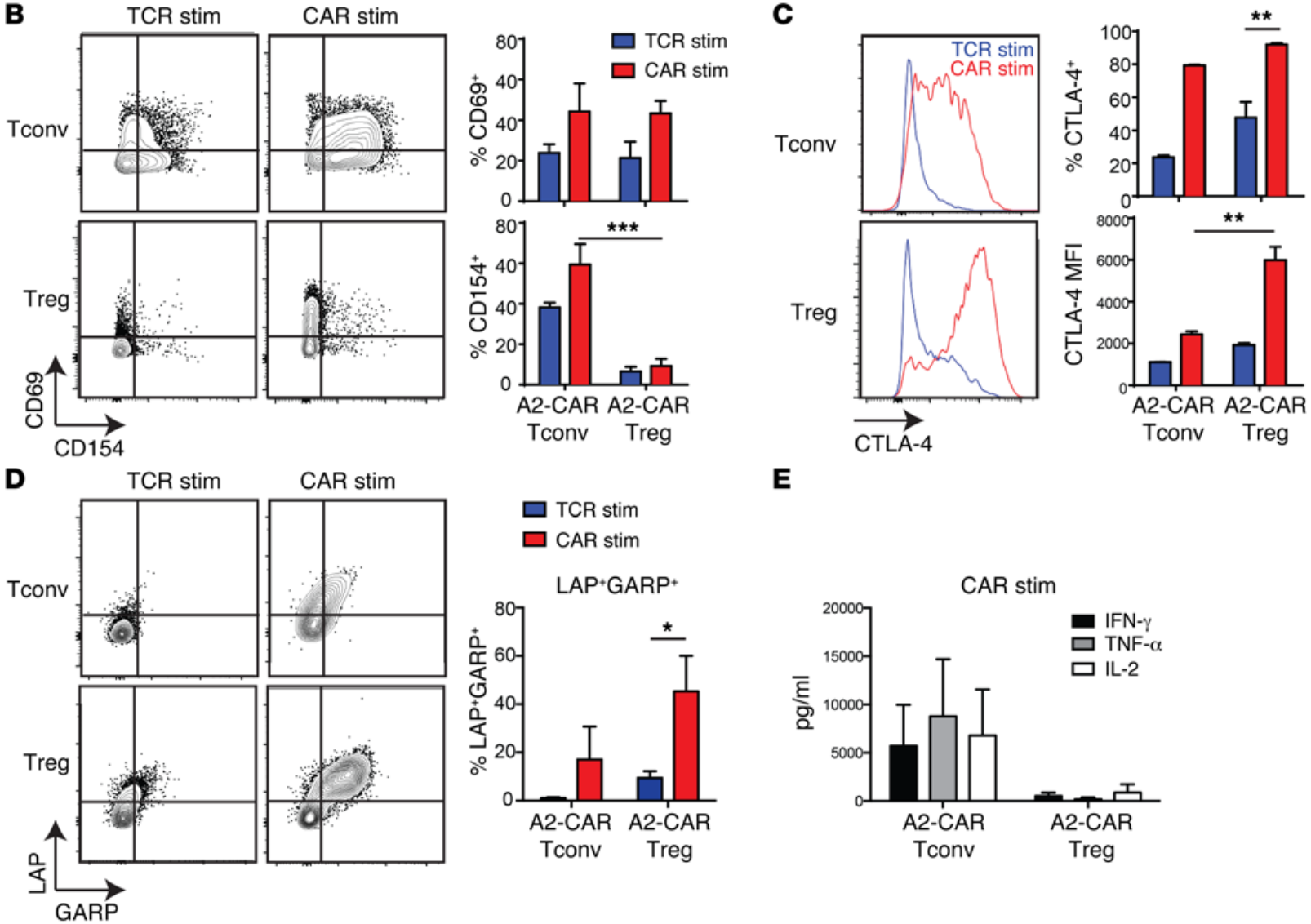

$\mathbf{E}$

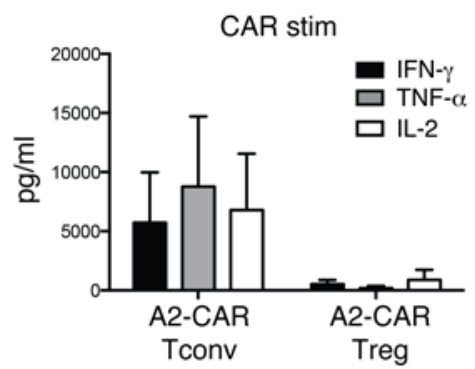

Figure 3. A2-CAR-mediated stimulation activates Tregs. (A) Signaling downstream of TCR or CAR stimulation was assessed in A2-CAR Tregs and Tconvs loaded with $\alpha$ CD3 (TCR) or $\alpha$ Myc (CAR) mAbs, which were then crosslinked by addition of $\alpha$ lgG. Cells were fixed at 0, 5, 10, and 20 minutes and stained for phosphorylated ZAP70 $(n=4)$. (B-D) A2-CAR Tregs or Tconvs were stimulated with K562.64 cells loaded with $\alpha$ CD3/28 mAbs (TCR stim) or expressing HLA-A2 (CAR stim) ( $n=4)$. After 1 day, T cells were assayed for expression of (B) CD69 and CD154, (C) CTLA-4, and (D) membrane-bound TCF- $\beta$ (LAP) and GARP. Gates were set based on unstimulated controls. (E) After 48 hours, cytokine production upon A2-CAR stimulation was measured by cytometric bead array $(n=2)$. Averaged data represent mean \pm SEM. Significance determined by 2 -way ANOVA. ${ }^{*} P<0.05 ;{ }^{* *} P<0.01 ;{ }^{* * *} P<0.001$.

HER2-CAR Tregs and HLA-A2 PBMCs. These data suggest that expression of A2-CAR enables Tregs to interact more specifically with HLA-A2-expressing cells.

To test the relative ability of A2-CAR Tregs to suppress T cell proliferation in response to allogeneic antigens, we set up mixed lymphocyte reactions (MLRs) in which either the responding $\mathrm{T}$ cells or the stimulating antigen-presenting cells (APCs) expressed HLA-A2. As shown in Figure 5, B and C, compared with HER2CAR Tregs, A2-CAR Tregs were significantly better able to suppress alloantigen-stimulated proliferation of $\mathrm{CD} 8^{+} \mathrm{T}$ cells regardless of whether HLA-A2 was expressed on the responder or the stimulator cells. These data show that expression of A2-CAR on Tregs results in antigen-specific suppression.
To further confirm the antigen specificity of the A2-CAR Tregs, we used an autologous system consisting of an EBV-transformed B cell line (with and without ectopic HLA-A2 expression), a tetanus toxoid-specific (TT-specific) $\mathrm{T}$ cell clone, and A2-CAR Tregs from an HLA-A2- individual (Supplemental Figure 2A). This system enabled the Tregs and responding $\mathrm{T}$ cell clone to be activated individually, without polyclonal anti-CD3 stimulation (i.e., TCR), allowing precise testing of antigen specificity. In the absence of HLA-A2, A2-CAR Tregs did not suppress TT-stimulated proliferation. In the presence of HLA-A2, only the A2-CAR, and not the HER2-CAR, Tregs suppressed proliferation. Finally, the presence of stimulated A2-CAR-expressing Tconvs enhanced proliferation. 

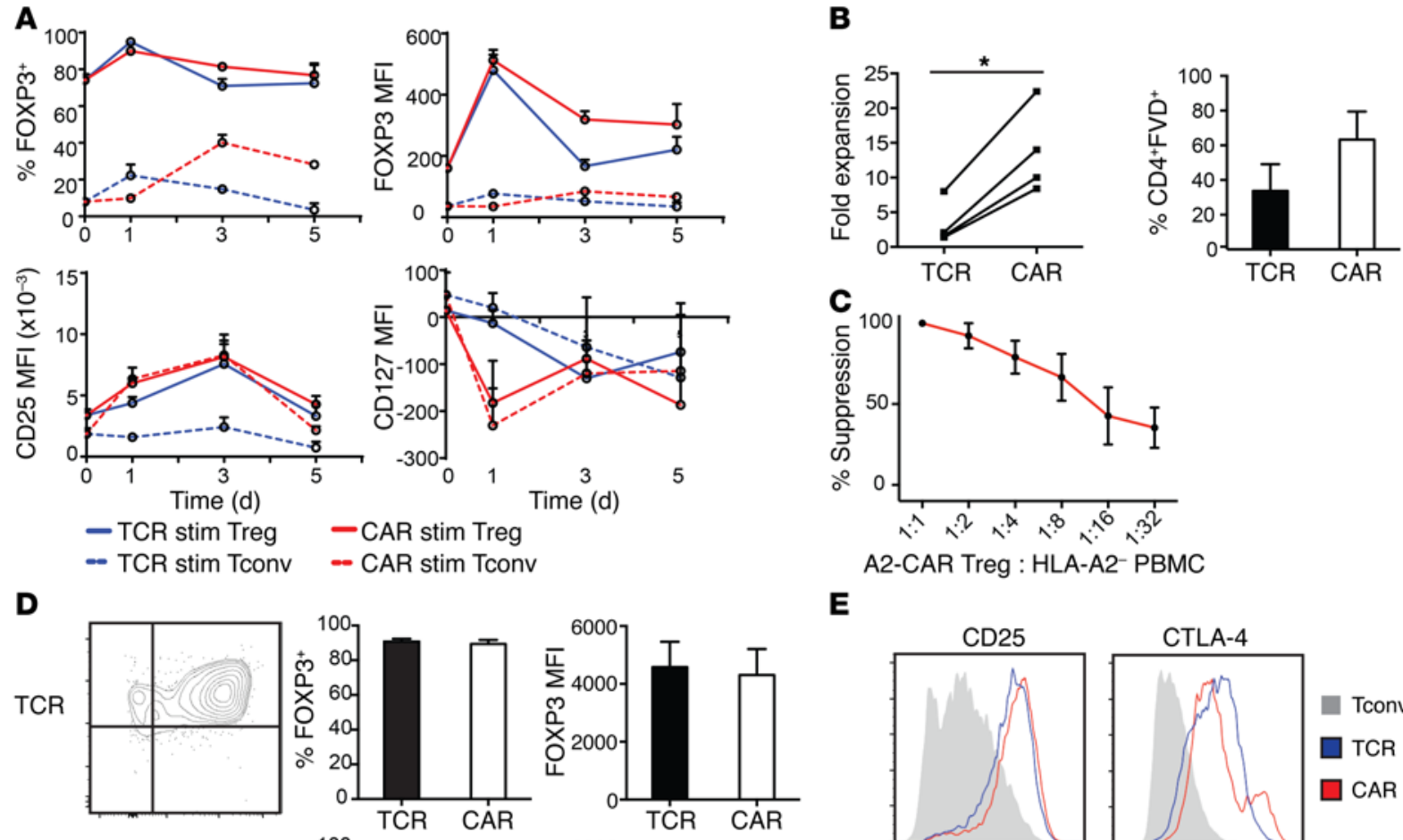

$\mathbf{E}$
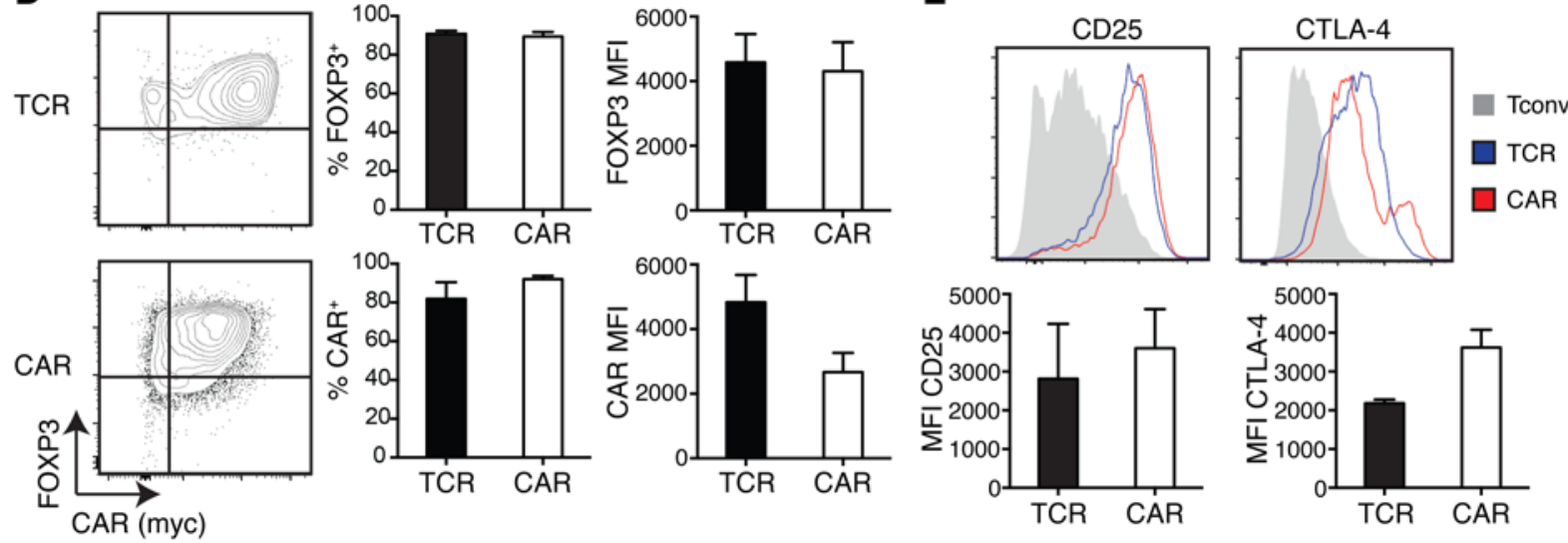

Figure 4. A2-CAR-mediated stimulation and expansion maintains a Treg phenotype. A2-CAR Tregs or Tconvs were stimulated with K562.64 cells loaded with $\alpha$ CD3/28 mAbs or expressing HLA-A2. (A) Expression of FOXP3, CTLA-4, CD25, and CD127 was assessed over 5 days on Tregs and Tconvs ( $n=4$ ). (B) Fold expansion based on cell counts at day 0 of restimulation and day 14, and viability of CAR- and TCR-expanded Tregs $(n=4)$. (C) Suppressive capacity of CAR-expanded A2-CAR Tregs when stimulated through endogenous TCR was assayed by titrated Treg ratios with HLA-A2- PBMCs stimulated with $\alpha$ CD3/28-coated beads $(n=3)$. (D) A2-CAR Treg phenotype after 2 weeks of expansion with TCR or CAR stimulation, both in the presence of IL-2. FOXP3 purity and CAR expression; representative plots on the left and summary plots on the right $(n=4)$. (E) Expression of CD25 and CTLA-4 on TCR-expanded or CAR-expanded Tregs (Tconv in gray, $n=4$ ). Averaged data represent mean \pm SEM. Significance determined by paired $t$ test. ${ }^{*} P<0.05$.

In vivo, A2-CAR Tregs would have the potential to receive simultaneous signals through both their endogenous TCRs and the ectopically expressed CAR. To mimic this possibility, HLA$\mathrm{A}^{+}$PBMCs were stimulated with $\alpha \mathrm{CD} 3 / 28$-coated beads in the absence or presence of A2-CAR- or HER2-CAR-expressing Tregs. A2-CAR Tregs, which received stimulation from both the CAR and the TCR (via $\alpha \mathrm{CD} 3 / 28$ beads), were as suppressive as HER2CAR Tregs (which only received TCR stimulation), demonstrating that combined CAR and TCR stimulation does not negatively affect suppressive function (Supplemental Figure 2B). A2-CARmediated suppressive activity was also found to require contact, as A2-CAR-stimulated Tregs were not suppressive in a Transwell suppression assay (Supplemental Figure 2C).

CAR-stimulated Tregs have minimal cytotoxic activity. CARs were originally developed in the context of cytolytic $\mathrm{T}$ cells, and there are some reports that human Tregs may also employ cytotoxicity as one of their mechanisms of suppression (40), so we next determined whether CAR stimulation might induce Tregs to kill their targets. $\mathrm{HLA}^{-\mathrm{A}^{+}}{ }^{+}$or HLA-A2 $2^{-}$K562 cells were cocultured with increasing numbers of A2-CAR Tregs or Tconvs, and after 24 hours, the proportion of K562 cells expressing active caspase 3 was measured as an indicator of cell death (Figure 6A). A2-CAR Tconvs robustly induced cell death in HLA-A2 ${ }^{+}$K562 cells (but not HLA-A2- K562 cells, data not shown). Although a small proportion $(<15 \%)$ of HLA$\mathrm{A} 2^{+} \mathrm{K} 562$ cells were positive for active caspase 3 when cocultured with high ratios of A2-CAR Tregs, A2-CAR Tregs mediated substantially less killing than Tconvs at all ratios tested (Figure 6B).

Possible Treg-mediated cytolytic activity was further tested using PBMCs as targets. Similar to data with K562 cells, coculture with A2-CAR Tconvs resulted in the presence of active caspase 3 in HLA-A2 ${ }^{+}$PBMCs. In contrast, in the presence of A2-CAR Tregs, there was negligible active caspase 3 (Figure 6C).

A2-CAR Tregs are superior to polyclonal Tregs at preventing xenogeneic GVHD mediated by HLA-A2+ $T$ cells. In order to test the functional capacity of A2-CAR Tregs in vivo, we used a mouse model in which human PBMCs engrafted into immunodeficient NOD/ SCID IL-2R $\gamma^{\text {null }}$ (NSG) mice cause xenogeneic GVHD (41). In these experiments, $1 \times 10^{7}$ PBMCs from an HLA-A2 ${ }^{+}$donor were inject- 
A

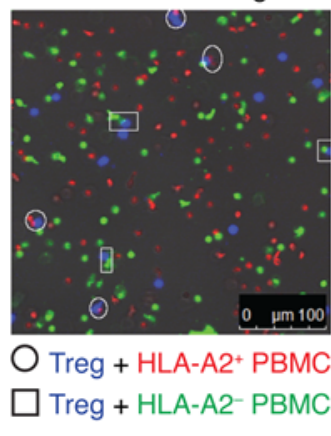

B

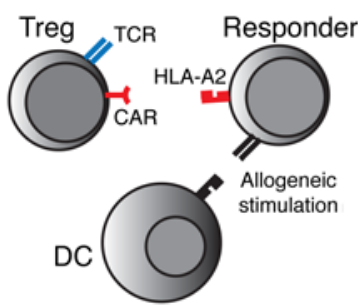

C

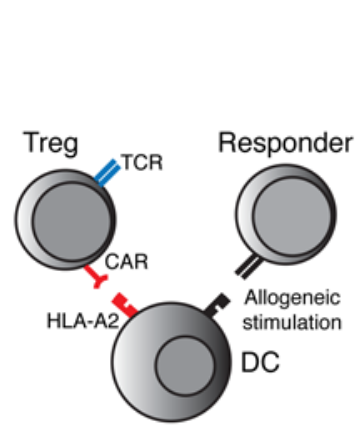

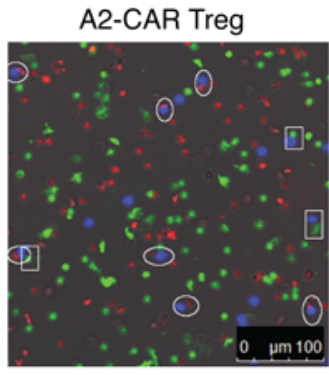

Ratio Treg : $\mathrm{CD}^{+}{ }^{+} \mathrm{HLA}-\mathrm{A} 2^{+}$responder 1:2.5

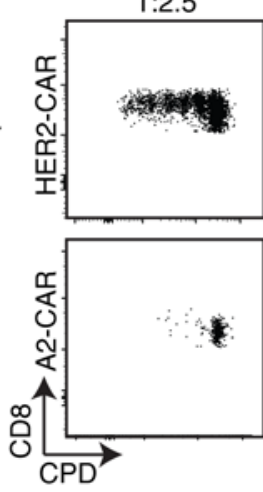

Ratio Treg : $\mathrm{CD}^{+} \mathrm{HLA}^{-\mathrm{A} 2^{-}}$responder $1: 2.5$

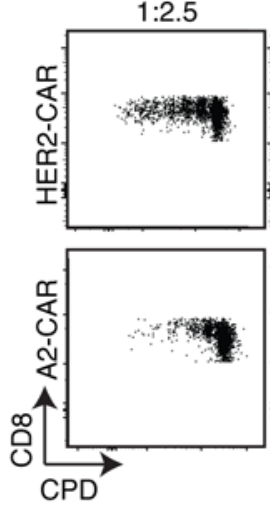

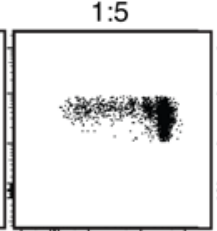
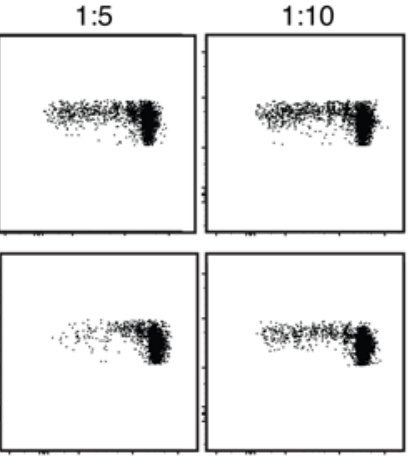

wards
No. of interactions per frame

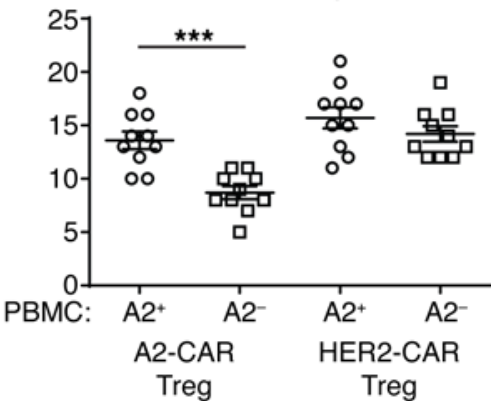

$1: 10$

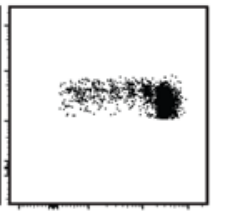

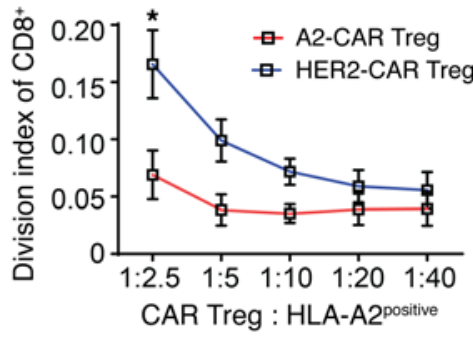

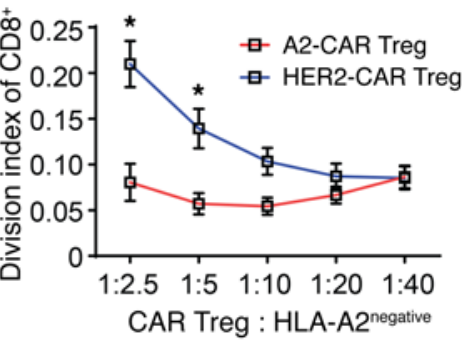

Figure 5. A2-CAR Tregs preferentially interact with HLA-A2 ${ }^{+}$PBMCs and are superior to polyclonal Tregs at suppressing alloantigen-stimulated proliferation in the presence of HLA-A2. (A) A2-CAR or HER2-CAR Tregs were cultured in a 3D collagen gel with a mixture of HLA-A2+ and HLA-A2- PBMCs, each labeled with a unique dye. Colocalization of CAR Tregs (blue) with HLA-A2+ (red, interaction indicated by circles) and HLA-A2- (green, interaction indicated by squares) PBMCs was observed over time by fluorescence microscopy. Representative data are shown on the left, with a summary of the number of interactions over 4 hours to the right. Data represent mean \pm SEM. Significance determined by 2-way ANOVA. (B and C) Suppressive capacity of transduced Tregs in allogeneic mixed leukocyte reactions was assayed by titrating the indicated ratios of A2-CAR or HER2-CAR Tregs into cultures with (B) HLA$\mathrm{A2}^{+} \mathrm{CD}^{+}$responders (labeled with CPD) stimulated by HLA-A2- monocyte-derived DCs or (C) HLA-A2- CD3+ responders (labeled with CPD) stimulated by $\mathrm{HLA}-\mathrm{A2} 2^{+} \mathrm{DC}$. Division index of gated $\mathrm{CD} 8^{+}$cells was determined after 96 hours. Averaged data (mean $\pm \mathrm{SEM}, n=4$ ) are shown. Significance determined by multiple $t$-tests with Holm-Šídák comparison. ${ }^{*} P<0.05 ;{ }^{* *} P<0.001$.

ed into irradiated NSG mice with or without the indicated type of Treg at a 1:1 or 2:1 ratio (i.e., $1 \times 10^{7}$ Tregs or $5 \times 10^{6}$ Tregs). Mice were monitored for up to 7 weeks by clinical score as described in Methods. Consistent with previous reports, control Tregs expressing the HER2-CAR, which would be stimulated via their endogenous TCR by xenogeneic antigens (but not through the CAR), significantly improved survival of mice when infused at a 1:1 ratio (Figure 7A). Importantly, mice receiving A2-CAR Tregs at either a 1:1 or 1:2 ratio were significantly better protected. A2-CAR Tregs improved survival (Figure 7A) and delayed onset of xenogeneic
GVHD (Figure 7B) in comparison with results in mice receiving HER2-CAR-expressing Tregs. Even when xenogeneic GVHD developed in mice that received A2-CAR Tregs, weight loss was less (Figure 7C) and disease progression was slower (Figure 7D).

Engraftment of human $\mathrm{T}$ cells and survival of the infused Tregs was monitored by weekly blood draws. As shown in Figure 7E, in comparison with mice receiving HER2-CAR Tregs, mice injected with A2-CAR Tregs had a lower absolute number of human HLA-A2 ${ }^{+} \mathrm{CD} 45^{+}$cells per $\mu$ l of blood. Lower absolute numbers of circulating HLA-A $2^{+} \mathrm{CD} 45^{+}$cells likely reflect the 
A
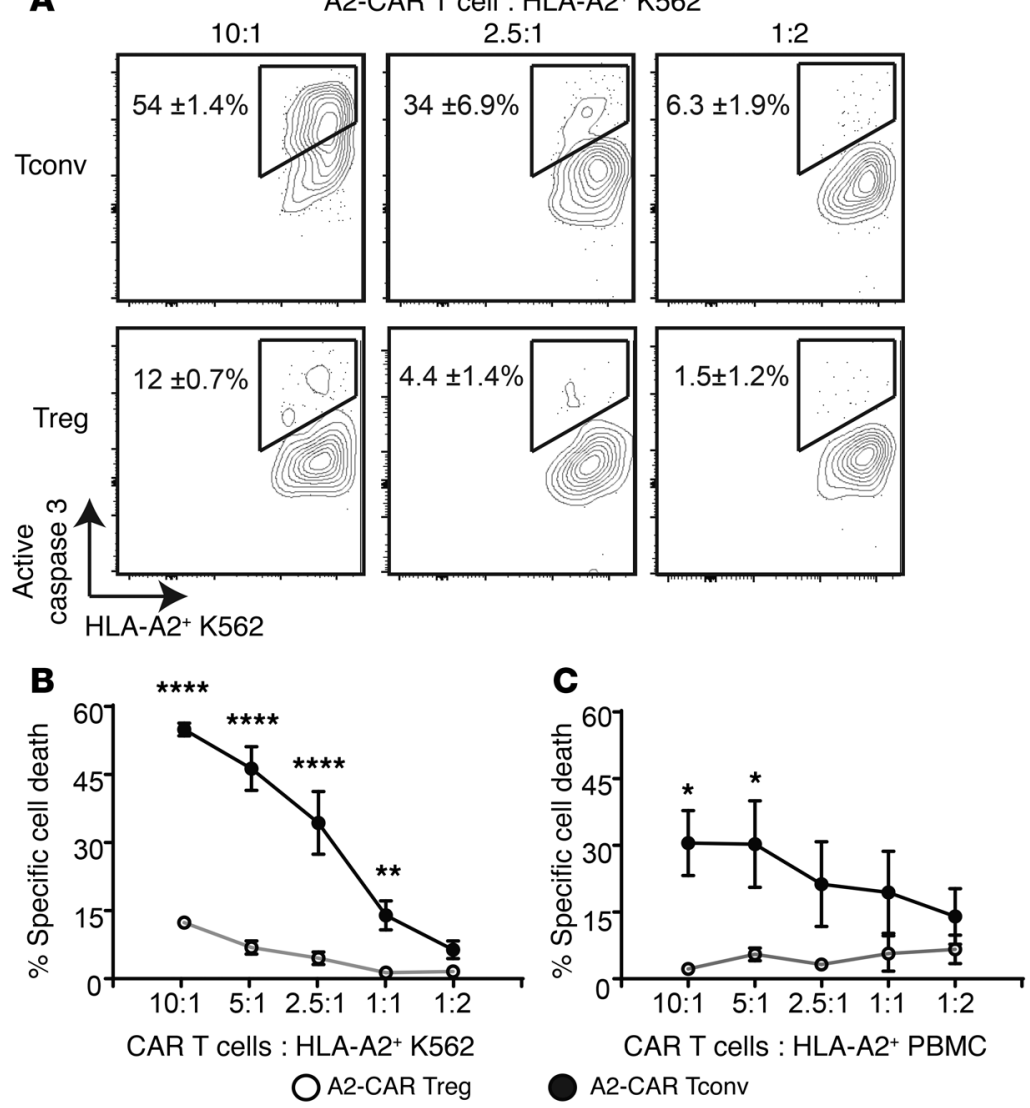

Figure 6. A2-CAR Tregs have minimal cytotoxic activity. A2-CAR Tregs or Tconvs were cocultured with $\mathrm{HLA}-\mathrm{A2} 2^{+} \mathrm{K} 562$ cells, which were labeled with $\mathrm{PKH} 26$ at the indicated ratios for 24 hours. (A) Representative plots of intracellular staining for active caspase 3. (B) Averaged cell death (mean \pm SEM, $n=4$ ). (C) Similar data were obtained when HLA-A2 ${ }^{+}$ PBMCs were used as targets (mean \pm SEM, $n=4$ ). Significance determined by multiple $t$ tests with Holm-Šídák comparison. ${ }^{*} P<0.05 ;{ }^{* *} P<0.01 ;{ }^{* * *} P<0.0001$.

failure of these cells to expand (as observed for mice coinjected with HER-2 Tregs), since the proportion of HLA-A2 ${ }^{+} \mathrm{CD} 45^{+}$cells was the same at days 7 and 14 and corresponded to expansion and persistence of A2-CAR Tregs in mouse blood (Figure 7, E and F). Notably, A2-CAR Tregs remained FOXP $3^{+}$and were detectable in circulation for twice as long as HER2-CAR Tregs. Similar to findings from in vitro data (Figure 4C), cells that received CAR stimulation in vivo had a lower MFI of CAR expression, but also had a significantly higher FOXP3 MFI (Figure 7G), likely reflecting in vivo activation.

In the xenogeneic GVHD model discussed above, HLA-A2 is expressed on the responding T cells, but in human HLA-A2 ${ }^{+}$recipients of hematopoietic stem cells or recipients of HLA-A2 $2^{+}$organs, HLA-A2 would be expressed ubiquitously on tissues. It was therefore important to exclude the possibility that the A2-CAR Tregs might cause tissue destruction. To test this possibility, HLA-A2 transgenic NSG mice were engrafted with HLA-A2- PBMCs in the absence or presence of A2-CAR Tregs or A2-CAR Tconvs. Mice were sacrificed at 2 different time points ( 2 and 4 weeks after cell infusion), and histological analysis was performed to measure immune cell infiltration and tissue integrity (Supplemental Figure 3). We found that mice injected with PBMCs, with or without
A2-CAR Tconvs, had substantial immune cell infiltration and tissue destruction. In contrast, mice receiving PBMCs and A2-CAR Tregs had less infiltration and most closely resembled the PBS control mice. Thus, injection of A2-CAR Tregs into mice that systemically express HLA-A2 does not result in tissue cytotoxicity, but rather activates their suppressive effect and limits PBMC-mediated tissue toxicity.

A2-CAR-stimulated Tregs require exogenous IL-2 for survival. A defining characteristic of human and mouse Tregs is in vitro anergy, i.e., their inability to proliferate in the absence of exogenous IL-2 $(39,42)$. We therefore asked whether CAR stimulation affected this phenotype, possibly overcoming a requirement for exogenous IL-2. We found that, in contrast with TCR, CAR could indeed stimulate IL-2-independent proliferation in short-term (3 day) assays, but addition of exogenous IL-2 significantly enhanced CAR- and TCR-stimulated cell division (Figure 8A). Beyond 3 days, however, in the absence of exogenous IL-2, CAR- and TCR-stimulated Tregs had a significant decrease in viability (Figure 8B). The inability of CAR stimulation to maintain long-term cell viability is consistent with the inability of the CAR to stimulate STAT5 phosphorylation (Figure 8C). Thus, although CAR stimulation results in potent Treg activation, such as that in unmodified Tregs, CAR-Tregs require exogenous IL-2 for long-term survival.

\section{Discussion}

In this study, we show that the specificity of human Tregs can be redirected toward a transplant-relevant antigen using a CAR. Expression of an A2-CAR in Tregs enables antigen-specific activation and proliferation that is stronger than that stimulated by the endogenous TCR. Despite this relatively strong CAR-mediated activation and/or expansion, A2-CAR Tregs retained high expression of FOXP3 and other Treg markers and demethylation of the TSDR, had preserved suppression function in vitro, and did not have significant cytolytic activity. Adoptive transfer experiments revealed that A2-CAR Tregs that received in vivo CAR stimulation were markedly better than Tregs that only received stimulation through the endogenous TCR at preventing xenogeneic GVHD. Collectively, these data show that human Tregs redirected to recognize a specific alloantigen are more effective at preventing GVHD than Tregs not thus directed, supporting the rationale for CAR modification of Tregs as a means of generating therapeutic antigen-specific cells.

Clinically applicable approaches to generating alloantigenspecific Tregs are currently limited to in vitro enrichment of alloreactive $\mathrm{T}$ cells following stimulation with allogeneic APCs. For example, alloantigen-specific Tregs can be sorted on the basis of allogeneic APC-stimulated expression of CD69 and CD71 (20) or expanded with CD40-stimulated allogeneic B cells (21). Cells generated by these methods are more potently suppressive in vitro than polyclonal Tregs and protect skin allografts better than polyclonal Tregs. Both these approaches, however, may suf- 

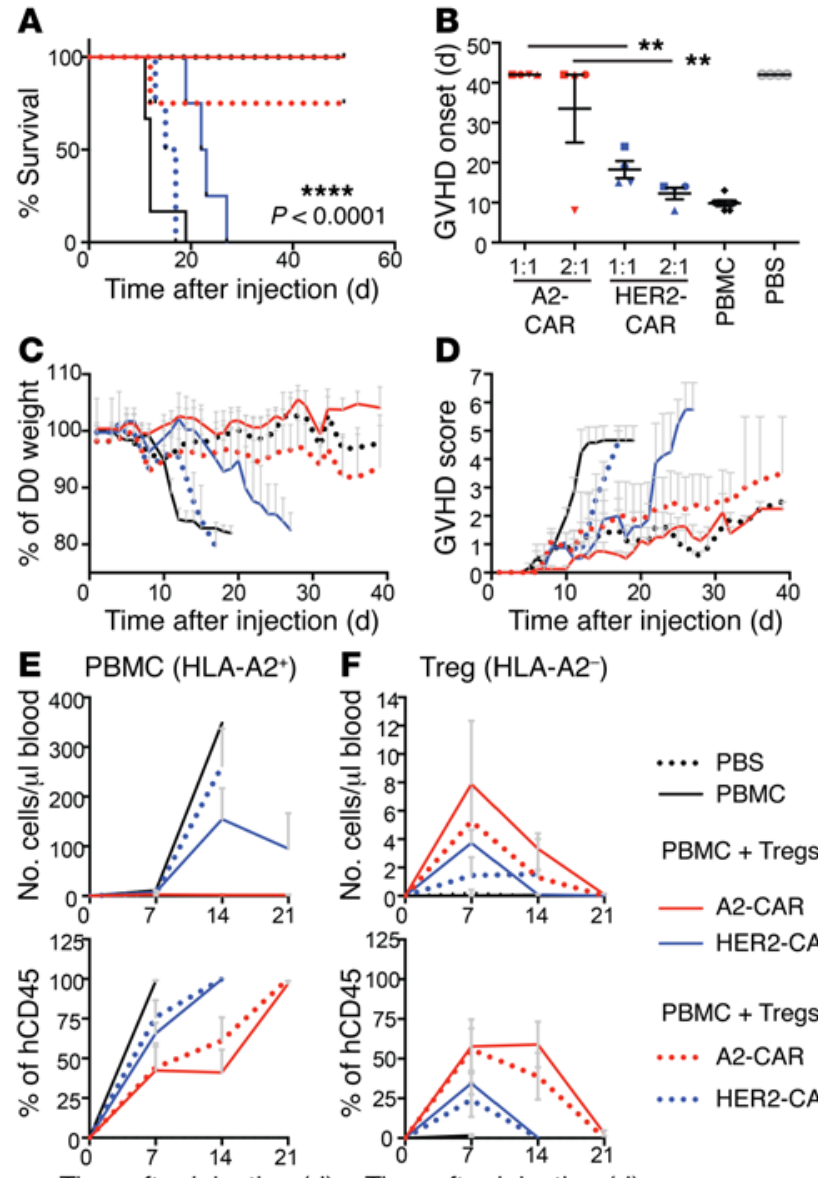

$$
\text { Time after injection (d) }
$$
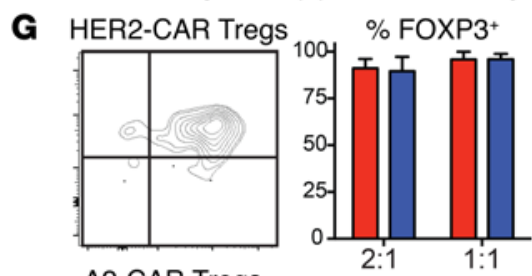

ection (d)

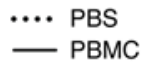

PBMC + Tregs (1:1 ratio)

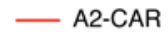

- HER2-CAR

PBMC + Tregs (2:1 ratio)

… A2-CAR

... HER2-CAR

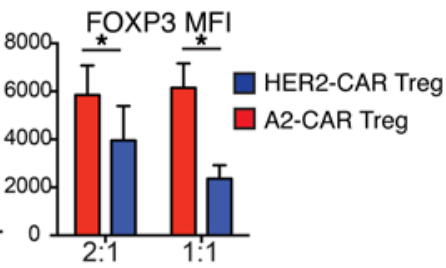

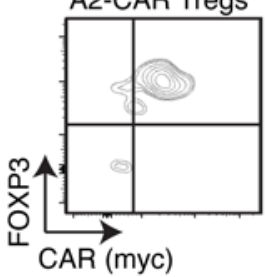

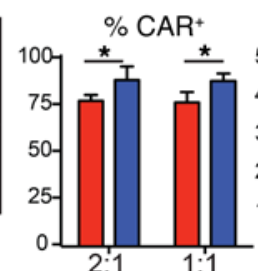

CAR MFI

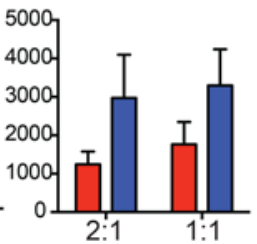

Figure 7. HLA-A2 CAR Tregs are superior to polyclonal Tregs at preventing xenogeneic GVHD. Irradiated NSG mice were injected with PBS $(n=4)$ or $1 \times 10^{7} \mathrm{HLA}-\mathrm{A} 2^{+}$ PBMCs alone $(n=6)$ or with $1 \times 10^{7}(1: 1)$ or $0.5 \times 10^{7}(1: 2)$ A2-CAR or HER2-CAR Tregs ( $n=4$ for each group). Two independent experiments were performed using Tregs generated from independent donors. Data shown are the combined results for all mice in 2 experiments. Blood was monitored every 7 days, and spleen engraftment was analyzed at end point (flow cytometry). (A) Survival curve and (B) day of GVHD onset. Each symbol shape represents a unique human donor from whose blood Tregs were made. (C) Percentage of weight loss relative to the start of experiment. (D) GVHD score. (E) Engraftment in numbers and proportion of PBMCs (HLA-A2 $)$ and (F) Tregs (HLA-A2-). (G) Representative plots and summary data for proportions and per-cell expression of FOXP3 and the relevant CAR in blood of mice receiving A2-CAR or HER2-CAR Tregs $(n=4)$. Data represent mean \pm SEM. Significance determined by log-rank test (A), 1-way ANOVA (B), and 2-way ANOVA (G). ${ }^{*} P<0.05 ;{ }^{*} P<0.01$.

fer from limitations of cell numbers, as the frequency of alloantigen-specific cells decreases with increasing MHC matching. Indeed, such approaches may not be feasible at all in the context of HSCT, since most or all of the HLA alleles are matched and in vitro expansion of alloantigen-specific cells decreases with increasing HLA matching $(21,43)$. Moreover, this method requires sufficient APCs from the donor, or recipient in the case of HSCT, which are not always available. An additional consideration is that expansion of alloantigen-specific Tregs by APC stimulation requires extensive in vitro expansion, which could lead to loss of FOXP3 (44) and, based on data from $\mathrm{CD} 8^{+} \mathrm{T}$ cells, decreased telomere length and, hence, in vivo survival (45). The use of CAR technology overcomes all of these limitations.

A2-CAR-expressing Tregs can be robustly activated by a single HLA mismatch. Targeting of Tregs with a CAR toward MHC class I, and specifically HLA-A2, provides several advantages: it bypasses the need for direct or indirect antigen presentation by MHC class II; MHC class I is broadly expressed on all tissues, so stimulation would not be dependent on the presence of professional APCs; and HLA-A2 is expressed by a substantial proportion of the population, leading to many HLA-A2-mismatched transplants. In the context of solid organ transplantation, expression of HLA-A2 on the organ would likely stimulate localization of A2-CAR Tregs to the transplant, a possibility supported by our observed preferential colocalization of A2-CAR Tregs with HLA-A2 ${ }^{+}$cells. In the context of GVHD, our experiments in HLA-A2-expressing NSG mice suggest that systemic expression of HLA-A2 may result in systemic activation of Treg suppression without consequent tissue damage.

Proof-of-concept work in mouse models has demonstrated the feasibility of redirecting the specificity of Tregs using CARs specific for model antigens. Expression of a trinitrophenol-specific CAR with a CD28 transmembrane domain and FC $\gamma \mathrm{R}$ signaling domains enabled mouse Tregs to home to the site of disease and protect from trinitrobenzene sulfonic acid-induced colitis (24, 25). Antigen-driven homing and protection from EAE was also observed in mouse $\mathrm{T}$ cells that were transduced with FOXP3 and a myelin oligodendrocyte glycoprotein-specific CAR containing domains from CD $3 \gamma$ and CD28 (26). There is also a previous report of expression of a CAR specific for carcinoembryonic antigen in human Tregs (27), but the low expression of FOXP3 and coproduction of IFN- $\gamma$ and IL-10 in these cells makes it difficult to draw any conclusions about the impact of CAR expression on Treg biology. Our study represents what we believe is a marked advance over these previous findings, as we report the creation of a human disease-relevant CAR and detailed data on the functionality of this protein in human Tregs.

An important consideration is how the presence of the CAR might affect the function of the endogenous TCR. We found that CAR expression does not abrogate the normal suppressive function of Tregs whether they 
A A2-CAR Treg
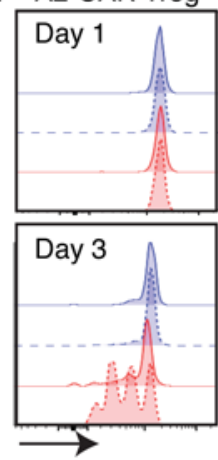

CPD

c
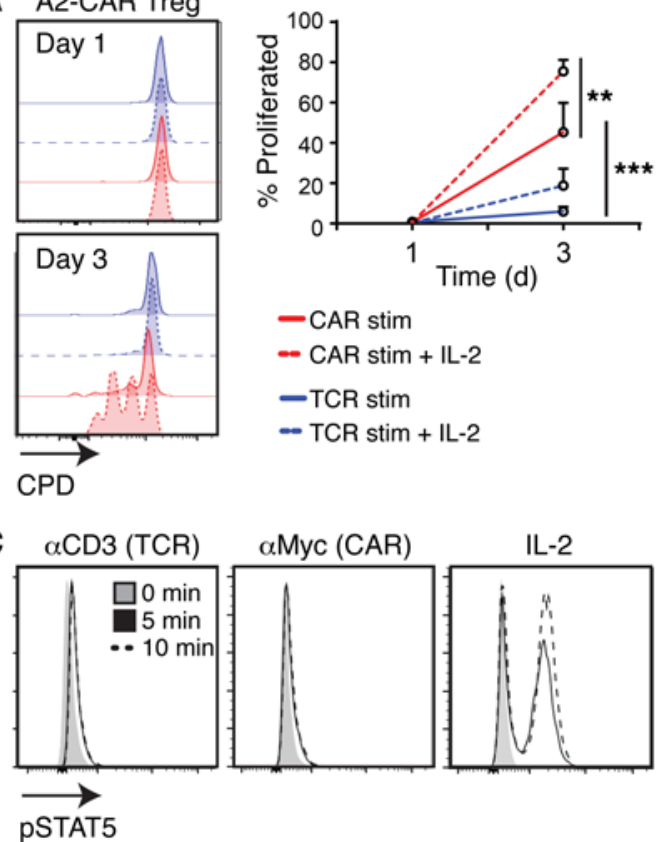

- CAR stim

- CAR stim + IL-2

-TCR stim

- TCR stim + IL-2
B

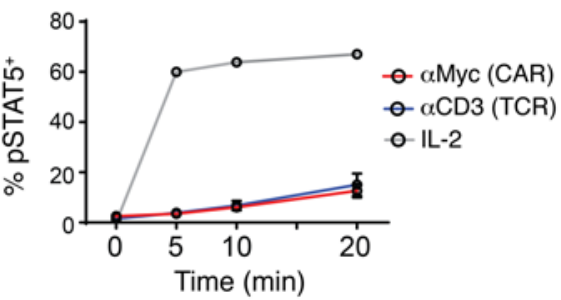

Figure 8. A2-CAR-stimulated Tregs require exogenous IL-2 for long-term survival. (A) A2-CAR Tregs or Tconvs were labeled with CPD, then stimulated with $\mathrm{K} 562.64$ cells loaded with $\alpha C D 3 / 28$ mAbs or expressing HLA-A2 in the absence or presence of $100 \mathrm{U} / \mathrm{ml}$ IL-2 for 5 days. Representative plots of $\mathrm{FVD}^{-} \mathrm{CD}^{+}$cells (left) and percentage of proliferated compared with unstimulated controls are summarized (right, $n=4)$. (B) The proportion of viable $\mathrm{CD}^{+} \mathrm{T}$ cells at day 5 was determined as the proportion of cells in the lymphocyte gate that were FVD-CD4 $(n=4)$. (C) STAT5 phosphorylation was assessed in A2-CAR Tregs stimulated via the TCR (with $\alpha \mathrm{CD} 3$ ), CAR ( $\alpha$ Myc), or IL-2. Cells were fixed at $0,5,10$, and 20 minutes, and the proportion of phospho-STAT5 positive cells was assessed $(n=3)$. Data represent mean \pm SEM. Significance determined by 1-way ANOVA (B) or 2-way ANOVA (A). ${ }^{* *} P<0.01 ;{ }^{* *} P<0.001$. are stimulated via the TCR or the CAR. This finding provides assurance that the "off-target" effects of CAR-expressing Tregs are not likely to be different from those of polyclonal Tregs and indeed should be minimized because the "on-target" effects can be obtained with smaller cell numbers.

Tregs are known to have intracellular signaling pathways distinct from those of Tconvs, with a specific requirement for low levels of PI3K activity to retain high FOXP3 expression (46). The secondgeneration format A2-CAR used here includes domains from CD28 and $\mathrm{CD} 3 \zeta$, both of which are necessary for Treg activation (47), suppressive capacity $(48,49)$, and homeostasis $(50,51)$. Although stimulation through the CAR did not cause abnormal signaling via ZAP70 or stimulate phosphorylation of STAT5, in short-term (2 to 3 days) assays, it did partially break the canonical anergy of Tregs in the absence of exogenous IL-2. Reversal of anergy, however, did not compromise lineage stability, as evidenced by stable expression of the expected Treg markers over time, including CD25, LAP, GARP, CTLA-4, and FOXP3, as well as suppressive function. In addition, exogenous IL-2 was necessary for long-term viability in vitro. Interestingly, activation of Tconv through the A2-CAR resulted in a Treglike phenotype, with high expression of CD25, CTLA-4, LAP, and GARP (see Figure 4), potentially related to the ability of tonic signaling from CD28 to lead to exhausted and less-potent Tconvs (52).

Since depletion of Tregs results in a return of inflammation in tolerized mice (53), long-term persistence may be essential for Treg therapy. In humans, infused Tregs have been observed in circulation for up to 14 days $(3,4)$, and in CAR T cell therapies, transferred cells are detectable in the blood for several weeks (54, 55). Although we observed A2-CAR Tregs in vivo for twice as long as polyclonal Tregs, they were not detectable in circulation after 2 weeks. Given that the HLA-A2 ${ }^{+}$PBMCs failed to expand in the presence of A2-CAR Tregs, it is possible that limited antigen, limited IL-2, or both were insufficient to sustain the expansion and survival of A2-CAR Tregs.
In conclusion, here we have demonstrated the feasibility of redirecting Tregs to a transplant-relevant antigen with a CAR and shown that CAR Tregs remain functionally and phenotypically stable in vitro and in vivo. This work provides what we believe is the first proof-of-concept that CAR Tregs have the potential to be used therapeutically. In the setting of transplantation, a bank of HLA-specific CARs could be used to overcome all of the limitations of current strategies to generate alloantigen-specific Tregs. These findings also set the stage for using CARs to redirect the antigen specificity of Tregs toward auto- and/or tissue-specific antigens for therapeutic use in autoimmunity.

\section{Methods}

A2-CAR generation. Variable regions of the Ig heavy and light chains were cloned from the anti-HLA-A2 BB7.2 hybridoma (ATCC) using published methods (56) and converted into a single-chain $\mathrm{Ab}(\mathrm{scFv})$. The scFv was fused to a Myc epitope tag in the extracellular region to enable cell-surface detection by flow cytometry, a stalk region from human $\mathrm{CD} 8 \alpha$, the transmembrane and intracellular domains of human $\mathrm{CD} 28$, and human $\mathrm{CD} 3 \zeta$, as described (33). The HER2-CAR2 and the A2-CAR were cloned into a lentiviral vector that encodes cell-surface expression of a truncated nerve-growth-factor (TrkA) receptor $(\triangle \mathrm{NGFR})$ as a marker. Surface expression was determined by flow cytometry with transiently transfected HEK 293T cells (Lipofectamine 2000, Life Technologies). Viral particles were produced as described (57).

Treg sorting, transduction, and expansion. $\mathrm{CD}^{+} \mathrm{T}$ cells were isolated from HLA-A2 ${ }^{-}$donors via RosetteSep (STEMCELL Technologies) and enriched for $\mathrm{CD}^{2} 5^{+}$cells (Miltenyi Biotec) prior to sorting into live $\mathrm{CD} 44^{+} \mathrm{CD} 45 \mathrm{RO}^{\text {lo }} \mathrm{CD} 45 \mathrm{RA}^{\text {hi }} \mathrm{CD} 25^{\text {hi }}$ Tregs and $\mathrm{CD} 4{ }^{+} \mathrm{CD} 45 \mathrm{RO}^{\text {lo }} \mathrm{CD} 45 \mathrm{RA}^{\text {hi }} \mathrm{CD} 25^{\text {lo }}$ Tconv), using a FACSAria II (BD Biosciences). Sorted $\mathrm{T}$ cells were stimulated with artificial APCs (aAPCs) loaded with $\alpha \mathrm{CD} 3 \mathrm{mAbs}$ as described (58) in 1,000 U/ml or $100 \mathrm{U} / \mathrm{ml}$ of IL-2, for Tregs or Tconvs, respectively. One day later, cells 
were transduced with lentivirus at an MOI of 10 virus particles/cell. At day $7, \Delta \mathrm{NGFR}^{+}$cells were purified with magnetic selection (Miltenyi Biotec), then restimulated with aAPCs as above and expanded for 6 to 7 days. To test effects of A2-mediated stimulation, Tregs were restimulated with irradiated (10 Gy) K562.64 cells (59), K562.64.HLA-A2 cells, or K562.64.HER2 cells (derived from K562.64 cells transduced with a lentivirus encoding HER2 and GFP) at a 1:2 (K562/T cell) ratio for 2 weeks in the presence of 1,000 U/ml IL-2.

Flow cytometry. For phenotypic analysis, cells were stained with fixable viability dye (FVD) (65-0865-14 and 65-0866-14, eBioscience) and for surface markers before fix/perm with FOXP3/Transcription Factor Staining Buffer Set (eBioscience), followed by staining for intracellular proteins. For analysis of cytokine production, cells were stimulated with $10 \mathrm{ng} / \mathrm{ml} \mathrm{PMA}$ and $500 \mathrm{ng} / \mathrm{ml}$ ionomycin, in the presence of brefeldin A $(10 \mu \mathrm{g} / \mathrm{ml})$ (all Sigma-Aldrich) for 4 hours. Samples were read on an LSRII or Fortessa (BD Biosciences) and results analyzed using FlowJo Software versions 8.7 and 10.0.6 (Tree Star).

Surface staining was performed for $\triangle$ NGFR (130-091-917, Miltenyi Biotec), HLA-A2 (551285, BD Biosciences), Myc (2233S, Cell Signalling), CD4 (46-0047-42, eBioscience), CD8 (48-0087-41, eBioscience or 555635, BD Biosciences), CD45RA (11-0458-73, eBioscience), CD45RO (48-0457-42, eBioscience), hCD45 (560777, BD Biosciences), mCD45 (25-0451-82, eBioscience), CD25 (120-001311, Miltenyi Biotec and 25-0259-42, eBioscience), LAP (25-9829-42, eBioscience), GARP (563958, BD Bioscience), CD69 (LMHCD6901, Caltag), CD154 (555702 BD Biosciences), and CD127 (48-1278-42, eBiosciences). HLA-A2 and HLA-A24 tetramers were a gift from $\mathrm{Ru}$ Tan (University of British Columbia).

Intracellular staining was performed for FOXP3 (12-4777-42, eBioscience), Helios (137223, Biolegend), CTLA-4 (555855 and 562743, BD Biosciences), IL-2 (559334, BD Biosciences), IFN- $\gamma$ (557844, BD Biosciences), and active caspase 3 (584098, BD Biosciences).

Microscopy. PBMCs were labeled with PKH26 or PKH67 (SigmaAldrich, PKH26GL-1KT and PKH67GL-1KT), and Tregs were labeled with cell proliferation dye (CPD) eFluor450 (eBiosciences, 65-084285 ) and then suspended in a $3 \mathrm{D}$ gel of $1.5 \%$ rat tail collagen type I (Ibidi) composed of $1 \times$ DMEM and 10\% FCS per the manufacturer's general 3D gel protocol. The cell suspension was pipetted into a $\mu$-Slide Chemotaxis ${ }^{3 \mathrm{D}}$ and allowed to polymerize for 30 minutes in a humidified incubator at $35^{\circ} \mathrm{C}$ and $5 \% \mathrm{CO}_{2}$ (Tokai Hit) on a Leica TCS SP8 confocal microscope. The outer chambers were then filled with $1 \times$ DMEM and images recorded using a $\times 10 / 0.30$ objective every 2 minutes for 3 hours. eFluor450, PKH67, and PKH26 were excited at $405 \mathrm{~nm}, 488 \mathrm{~nm}$, and $561 \mathrm{~nm}$, and the fluorescence emission was collected at $415-470 \mathrm{~nm}, 495-525 \mathrm{~nm}$, and $570-650 \mathrm{~nm}$, respectively. The number of interactions between CAR-Tregs and either HLA-A2 ${ }^{+}$ or HLA-A2 cells was quantified every 20 minutes. Cells that did not move were excluded from the analysis. The total numbers of each labeled cell type per field of view were counted using the analyze particles function in ImageJ (http://imagej.nih.gov/ij/).

TSDR analysis. DNA from frozen T cell pellets of male donors was isolated with the DNeasy Blood and Tissue Kit (QIAGEN) and bisulfite converted with the EZ Direct Kit (Zymo Research). PCR of BisDNA was performed with the Human FOXP3 Kit (Epigen DX) and prepared for pyrosequencing using PyroMark buffers (QIAGEN), then assayed on a Biotage PyroMark Q96 MD pyrosequencer (QIAGEN). Results were calculated with Pyro Q-CpG software (Biotage).
Proliferation, activation, cytokine production, and antigen-nonspecific suppression. To assess proliferation and activation, $\mathrm{T}$ cell lines were labeled with CPD (65-0840-85 or 65-0842-85, eBiosciences) and stimulated with K562.64 cells loaded by 1 hour of preincubation with $\alpha \mathrm{CD} 3$ and $\alpha 28 \mathrm{mAbs}(1 \mu \mathrm{g} / \mathrm{ml}$ each), K562.64.HLA-A2 cells, or at a 1:2 (K562:T cell) ratio (K562.64 cells, a gift from James Riley, University of Pennsylvania, Philadelphia, Pennsylvania, USA, which were then transduced to express HLA-A2). Staining of CD154 was performed in culture for 6 hours prior to analysis. Suppression was assessed with allogeneic HLA-A2 ${ }^{-}$or HLA-A2 ${ }^{+}$PBMCs labeled with CPD450 and stimulated via $\alpha C D 3 / 28$-coated beads (Invitrogen) at a 1:8 or 1:16 bead to PBMC ratio for 96 hours. Percentage of suppression of $\mathrm{CD} 8^{+}$cells was calculated using division index (DI) as follows: $(100-[(D I P B M C s+t e s t) /(D I$ PBMCs) $] \times 100)$. For Transwell assays, CAR T cells were cocultured with HLA-A2 ${ }^{+} \mathrm{K} 562$ cells at a 1:1 ratio in an insert with a $4-\mu \mathrm{m}$ pore membrane (Millipore).

To measure cytokine production, $\mathrm{T}$ cell lines were stimulated with the indicated K562 cells ( 1 K562:2 T cells) for 48 hours. Supernatants were collected and cytokine concentration was determined by the Human Th1/Th2/Th17 Cytokine Kit (BD Biosciences) and analyzed by FCAP Array Software v1.01 (Soft Flow).

Cytotoxicity assays. HLA-A2 ${ }^{+} \mathrm{K} 562$ cells or PBMCs were labeled with PKH26 (Sigma-Aldrich, PKH26GL-1KT) and cocultured with A2-CARexpressing cells at the indicated ratios for 24 hours. Activation of caspase 3 in $\mathrm{PKH} 26^{+}$cells was determined by flow cytometry and percentage-specific cell death was calculated by subtracting the percentage of cells with active caspase 3 in cultures with no CAR Tregs/Tconvs as described (60).

Suppression of MLRs and TT-specific T cells. Adherent cells from HLA-A2 ${ }^{+}$or HLA-A2 ${ }^{-}$PBMCs from healthy donors were differentiated into monocyte-derived DCs as described (61). For MLRs, HLA-A2 ${ }^{+}$or HLA-A2- $\mathrm{CD}^{+}$responder $\mathrm{T}$ cells were labeled with CPD eF450 (eBiosciences, 65-0842-85); then $1 \times 10^{5} \mathrm{CD}^{+}$responder $\mathrm{T}$ cells were stimulated with $5 \times 10^{4}$ HLA-A2-mismatched DCs, with increasing ratios of A2-CAR or HER2-CAR Tregs. After 4 days, division of CD8 ${ }^{+} \mathrm{T}$ cells was measured by flow cytometry.

For suppression of TT-specific proliferation, TT-specific CD $4^{+} \mathrm{T}$ clones were isolated from an HLA-A2 individual as described (62). An EBV cell line from the same donor was transduced with HLA-A2 using lentivirus. EBV cell lines were pulsed overnight with $50 \mu \mathrm{g} / \mathrm{ml}$ of TT ( $5 \mu \mathrm{g} / \mathrm{ml}$, Enzo Life Science, ALX-630-108), irradiated at 150 Gy, and cocultured with CPD-labeled TT-specific CD $4^{+} \mathrm{T}$ clones in the absence of presence of CAR-expressing Tregs of Tconvs. Proliferation was determined after 4 days, and percentage of suppression of TT-specific clones was calculated using percentage of proliferation as follows: $(100-[(\%$ proliferated $\mathrm{TT}+$ test $) /(\%$ proliferated TT alone) $] \times 100)$.

In vivo experiments. The 8- to 12-week-old female NSG mice (The Jackson Laboratory, bred in house) received whole-body irradiation (150 cGy, RS-2000 Pro Biological System) 1 day before injection of $1 \times$ $10^{7}$ HLA-A2 ${ }^{+}$PBMCs with or without $1 \times 10^{7}$ or $0.5 \times 10^{7}$ of the indicated type of Tregs. Saline-injected mice served as controls. GVHD was scored based on weight, fur texture, posture, activity level, and skin integrity, with 0 to 2 points per category as described $(63,64)$. GVHD scoring was performed by 2 blinded investigators. Peripheral blood from the saphenous vein was centrifuged; then erythrocytes were lysed and leukocytes were measured by flow cytometry, and plasma was aspirated and frozen at $-80^{\circ} \mathrm{C}$ until use. 
For experiments with A2-NSG mice, 8- to 12-week-old female NOD.Cg-Prkdc ${ }^{\text {scid }}$ Il2rg ${ }^{\text {tm1 }}$ Wjl Tg(HLA-A/H2-D/B2M)1Dvs/SzJ mice (The Jackson Laboratory) were engrafted with HLA-A2- PBMCs and $\mathrm{T}$ cell populations as indicated. Mice were sacrificed at indicated time points, and tissue samples for histology were fixed in 10\% formalin and embedded in paraffin. Sections were stained with $\mathrm{H} \& \mathrm{E}$, and pictures were acquired on an Olympus-BX61 using Image-Pro 6.2.

Statistics. Analysis was performed using Prism 6 software (GraphPad). $P<0.05$ was considered significant. Significance of fold expansion was determined by a 2 -tailed, paired $t$ test. Significance of survival was determined by log-rank (Mantel-Cox) test. Significance of GVHD onset was determined by ordinary 1-way ANOVA with Turkey's multiple comparisons test. All other significance was determined by 2-way ANOVA with Sidak's multiple comparisons test or multiple $t$ tests with Holm-Šídák comparison.

Study approval. For human cells, healthy volunteers gave written informed consent according to protocols approved by the University of British Columbia Clinical Research Ethics Board and Canadian Blood Services. Animal protocols were approved by the University of British Columbia Animal Care Committee.

\section{Author contributions}

KGM, REH, QH, JG, and DSL designed and performed experiments and analyzed data. PCO contributed essential reagents and also contributed to experimental design and data interpretation. KGM and MKL wrote the manuscript, which was critically reviewed by PCO and RB. RB and MKL secured funding. MKL conceived of and directed the research, analyzed data, and had overall oversight over the manuscript.

\section{Acknowledgments}

This work was supported by the Canadian Cancer Society Research Institute (to R. Broady and M.K. Levings) and the BC Transplant Research Foundation (to M.K. Levings). M.K. Levings, K.G. MacDonald, and R.E. Hoeppli receive salary awards from the Child \& Family Research Institute. In addition, K.G. MacDonald and R.E. Hoeppli were supported by a graduate studentship from the Canadian Institute for Health Research Training Program in Transplantation. We thank Jonathan Bramson for ongoing support and discussions and John Schrader and Welson Wang for technical support in cloning the scFv. We also thank Lisa Xu for excellent flow cytometry support and Scott Patterson for assisting with in vivo experiments. We are grateful to Canadian Blood Services and donors for providing research samples for completion of this project.

Address correspondence to: Megan K. Levings, A4-186, 950 West 28th Ave., Vancouver, British Columbia, Canada, V5Z 4H4. Phone: 604.875.2000, ext.4686; E-mail:mlevings@mail.ubc.ca.
1. Trzonkowski P, et al. First-in-man clinical results of the treatment of patients with graft versus host disease with human ex vivo expanded $\mathrm{CD} 4^{+} \mathrm{CD} 25^{+} \mathrm{CD} 127^{-} \mathrm{T}$ regulatory cells. Clin Immunol. 2009;133(1):22-26.

2. Di Ianni M, et al. Tregs prevent GVHD and promote immune reconstitution in HLA-haploidentical transplantation. Blood. 2011;117(14):3921-3928.

3. Brunstein CG, et al. Infusion of ex vivo expanded $T$ regulatory cells in adults transplanted with umbilical cord blood: safety profile and detection kinetics. Blood. 2011;117(3):1061-1070.

4. Marek-Trzonkowska N, et al. Administration of $\mathrm{CD} 4{ }^{+} \mathrm{CD} 25^{\text {high }} \mathrm{CD} 127^{-}$regulatory $\mathrm{T}$ cells preserves beta-cell function in type 1 diabetes in children. Diabetes Care. 2012;35(9):1817-1820.

5. Bluestone JA, et al. Type 1 diabetes immunotherapy using polyclonal regulatory T cells. Sci Transl Med. 2015;7(315):315ra189.

6. Brunstein CG, et al. Adoptive transfer of umbilical cord blood-derived regulatory $\mathrm{T}$ cells and early viral reactivation. Biol Blood Marrow Transplant. 2013;19(8):1271-1273.

7. Green EA, Choi YW, Flavell RA. Pancreatic lymph node-derived CD4(+)CD25(+) Treg cells: Highly potent regulators of diabetes that require TRANCE-RANK signals. Immunity. 2002;16(2):183-191.

8. Tang $Q$, et al. In vitro-expanded antigen-specific regulatory $\mathrm{T}$ cells suppress autoimmune diabetes. JExp Med. 2004;199(11):1455-1465.

9. Tarbell KV, Yamazaki S, Olson K, Toy P, Steinman RM. CD $25^{+} \mathrm{CD} 4^{+} \mathrm{T}$ cells, expanded with dendritic cells presenting a single autoantigenic peptide, suppress autoimmune diabetes. J Exp Med. 2004;199(11):1467-1477.

10. Masteller EL, Warner MR, Tang Q, Tarbell KV,
McDevitt H, Bluestone JA. Expansion of functional endogenous antigen-specific $\mathrm{CD} 4{ }^{+} \mathrm{CD} 25^{+}$ regulatory $\mathrm{T}$ cells from nonobese diabetic mice. JImmunol. 2005;175(5):3053-3059.

11. Tarbell KV, et al. Dendritic cell-expanded, isletspecific $\mathrm{CD}^{+} \mathrm{CD} 25^{+} \mathrm{CD}^{+} 2 \mathrm{~L}^{+}$regulatory T cells restore normoglycemia in diabetic NOD mice. JExp Med. 2007;204(1):191-201.

12. Stephens LA, Malpass KH, Anderton SM. Curing CNS autoimmune disease with myelin-reactive Foxp3 $3^{+}$Treg. Eur JImmunol. 2009;39(4):1108-1117.

13. Tsang JY, et al. Conferring indirect allospecificity on $\mathrm{CD} 4{ }^{+} \mathrm{CD} 25^{+}$Tregs by TCR gene transfer favors transplantation tolerance in mice. J Clin Invest. 2008;118(11):3619-3628.

14. Sanchez-Fueyo A, et al. Specificity of CD $4{ }^{+} \mathrm{CD} 25$ regulatory $\mathrm{T}$ cell function in alloimmunity. J Immunol. 2006;176(1):329-334.

15. Nishimura E, Sakihama T, Setoguchi R, Tanaka $\mathrm{K}$, Sakaguchi S. Induction of antigen-specific immunologic tolerance by in vivo and in vitro antigen-specific expansion of naturally arising Foxp $3^{+} \mathrm{CD} 25^{+} \mathrm{CD} 4^{+}$regulatory T cells. Int Immunol. 2004;16(8):1189-1201.

16. Joffre $\mathrm{O}$, et al. Prevention of acute and chronic allograft rejection with $\mathrm{CD} 4^{+} \mathrm{CD} 25^{+} \mathrm{Foxp}^{+}$regulatory T lymphocytes. Nat Med. 2008;14(1):88-92.

17. Golshayan D, Jiang S, Tsang J, Garin MI, Mottet $\mathrm{C}$, Lechler RI. In vitro-expanded donor alloantigen-specific $\mathrm{CD} 4{ }^{+} \mathrm{CD} 25^{+}$regulatory T cells promote experimental transplantation tolerance. Blood. 2007;109(2):827-835.

18. Trenado A, et al. Ex vivo-expanded $\mathrm{CD} 4^{+} \mathrm{CD} 25^{+}$ immunoregulatory $\mathrm{T}$ cells prevent graftversus-host-disease by inhibiting activation/ differentiation of pathogenic T cells. J Immunol. 2006;176(2):1266-1273
19. Verginis P, McLaughlin KA, Wucherpfennig KW, von Boehmer H, Apostolou I. Induction of antigen-specific regulatory $\mathrm{T}$ cells in wild-type mice: visualization and targets of suppression. Proc Natl Acad Sci U S A. 2008;105(9):3479-3484.

20. Sagoo P, Ali N, Garg G, Nestle FO, Lechler RI, Lombardi G. Human regulatory T cells with alloantigen specificity are more potent inhibitors of alloimmune skin graft damage than polyclonal regulatory T cells. Sci Transl Med. 2011;3(83):83ra42.

21. Putnam AL, et al. Clinical grade manufacturing of human alloantigen-reactive regulatory $\mathrm{T}$ cells for use in transplantation. Am J Transplant. 2013;13(11):3010-3020.

22. June CH, Riddell SR, Schumacher TN. Adoptive cellular therapy: a race to the finish line. Sci Transl Med. 2015;7(280):280ps7.

23. Gill S, June $\mathrm{CH}$. Going viral: chimeric antigen receptor T-cell therapy for hematological malignancies. Immunol Rev. 2015;263(1):68-89.

24. Elinav E, Adam N, Waks T, Eshhar Z. Amelioration of colitis by genetically engineered murine regulatory $\mathrm{T}$ cells redirected by antigenspecific chimeric receptor. Gastroenterology. 2009;136(5):1721-1731.

25. Elinav E, Waks T, Eshhar Z. Redirection of regulatory $\mathrm{T}$ cells with predetermined specificity for the treatment of experimental colitis in mice. Gastroenterology. 2008;134(7):2014-2024.

26. Fransson M, et al. CAR/FoxP3-engineered T regulatory cells target the CNS and suppress EAE upon intranasal delivery. J Neuroinflammation. 2012;9:112.

27. Hombach AA, Kofler D, Rappl G, Abken H. Redirecting human $\mathrm{CD} 4{ }^{+} \mathrm{CD} 25^{+}$regulatory T cells from the peripheral blood with pre-defined target 
specificity. Gene Ther. 2009;16(9):1088-1096.

28. Blat D, Zigmond E, Alteber Z, Waks T, Eshhar Z Suppression of murine colitis and its associated cancer by carcinoembryonic antigen-specific regulatory T cells. Mol Ther. 2014;22(5):1018-1028.

29. Collins MM, et al. The relative frequencies of HLA-DRB $1^{*} 01$ alleles in the major US populations. Tissue Antigens. 2000;55(1):48-52.

30. Park M, Seo JJ. Role of HLA in hematopoietic stem cell transplantation. Bone Marrow Res. 2012;2012:680841.

31. Sadelain M, Brentjens R, Riviere I. The promise and potential pitfalls of chimeric antigen receptors. Curr Opin Immunol. 2009;21(2):215-223.

32. Bour-Jordan H, Bluestone JA. Regulating the regulators: costimulatory signals control the homeostasis and function of regulatory $\mathrm{T}$ cells. Immunol Rev. 2009;229(1):41-66.

33. Sadelain M, Brentjens R, Riviere I. The basic principles of chimeric antigen receptor design. Cancer Discov. 2013;3(4):388-398.

34. Albrecht H, Denardo GL, Denardo SJ. Monospecific bivalent scFv-SH: effects of linker length and location of an engineered cysteine on production, antigen binding activity and free $\mathrm{SH}$ accessibility. JImmunol Methods. 2006;310(1-2):100-116.

35. Gu X, et al. Molecular modeling and affinity determination of scFv antibody: proper linker peptide enhances its activity. Ann Biomed Eng. 2010;38(2):537-549.

36. Hoffmann P, et al. Only the CD45RA ${ }^{+}$subpopulation of $\mathrm{CD} 4^{+} \mathrm{CD} 25^{\text {high }} \mathrm{T}$ cells gives rise to homogeneous regulatory T-cell lines upon in vitro expansion. Blood. 2006;108(13):4260-4267.

37. Frigault MJ, et al. Identification of chimeric antigen receptors that mediate constitutive or inducible proliferation of T cells. Cancer Immunol Res. 2015;3(4):356-367.

38. Rainbow DB, et al. Epigenetic analysis of regulatory $\mathrm{T}$ cells using multiplex bisulfite sequencing. Eur J Immunol. 2015;45(11):3200-3203.

39. Levings MK, Sangregorio R, Roncarolo MG. Human cd25(+)cd4(+) t regulatory cells suppress naive and memory $\mathrm{T}$ cell proliferation and can be expanded in vitro without loss of function. J Exp Med. 2001;193(11):1295-1302.

40. Grossman WJ, Verbsky JW, Barchet W, Colonna M, Atkinson JP, Ley TJ. Human T regulatory cells can use the perforin pathway to cause autologous target cell death. Immunity. 2004;21(4):589-601.

41. Hippen KL, et al. Massive ex vivo expansion of human natural regulatory $\mathrm{T}$ cells ( $\mathrm{T}(\mathrm{regs})$ ) with minimal loss of in vivo functional activity. Sci Transl Med. 2011;3(83):83ra41.

42. Thornton AM, Shevach EM. CD $4^{+} \mathrm{CD} 25^{+}$immunoregulatory $\mathrm{T}$ cells suppress polyclonal $\mathrm{T}$ cell activation in vitro by inhibiting interleukin 2 production. J Exp Med.1998;188(2):287-296.

43. Mickelson EM, Guthrie LA, Etzioni R, Anasetti C, Martin PJ, Hansen JA. Role of the mixed lymphocyte culture (MLC) reaction in marrow donor selection: matching for transplants from related haploidentical donors. Tissue Antigens. 1994;44(2):83-92.

44. Hoffmann P, et al. Loss of FOXP3 expression in natural human $\mathrm{CD} 4{ }^{+} \mathrm{CD} 25^{+}$regulatory $\mathrm{T}$ cells upon repetitive in vitro stimulation. Eur JImmunol. 2009;39(4):1088-1097.

45. Rosenberg SA, Dudley ME. Adoptive cell therapy for the treatment of patients with metastatic melanoma. Curr Opin Immunol. 2009;21(2):233-240.

46. Han JM, Patterson SJ, Levings MK. The role of the PI3K signaling pathway in CD4(+) T cell differentiation and function. Front Immunol. 2012;3:245.

47. Huynh A, Zhang R, Turka LA. Signals and pathways controlling regulatory $\mathrm{T}$ cells. Immunol Rev. 2014;258(1):117-131.

48. Schmidt AM, et al. Regulatory T cells require TCR signaling for their suppressive function. JImmunol. 2015;194(9):4362-4370.

49. Zhang R, Huynh A, Whitcher G, Chang J, Maltzman JS, Turka LA. An obligate cellintrinsic function for CD28 in Tregs. JClin Invest. 2013;123(2):580-593.

50. Vahl JC, et al. Continuous $\mathrm{T}$ cell receptor signals maintain a functional regulatory $\mathrm{T}$ cell pool. Immunity. 2014;41(5):722-736.

51. Levine AG, Arvey A, Jin W, Rudensky AY. Continuous requirement for the TCR in regulatory $\mathrm{T}$ cell function. Nat Immunol. 2014;15(11):1070-1078.

52. Long AH, et al. 4-1BB costimulation ameliorates T cell exhaustion induced by tonic signaling of chimeric antigen receptors. Nat Med. 2015;21(6):581-590.

53. Kendal AR, et al. Sustained suppression by Foxp $3^{+}$regulatory $\mathrm{T}$ cells is vital for infec- tious transplantation tolerance. J Exp Med. 2011;208(10):2043-2053.

54. Maus MV, Grupp SA, Porter DL, June CH. Antibody-modified T cells: CARs take the front seat for hematologic malignancies. Blood. 2014;123(17):2625-2635.

55. Maude SL, et al. Chimeric antigen receptor $\mathrm{T}$ cells for sustained remissions in leukemia. $N$ Engl JMed. 2014;371(16):1507-1517.

56. Babcook JS, Leslie KB, Olsen OA, Salmon RA, Schrader JW. A novel strategy for generating monoclonal antibodies from single, isolated lymphocytes producing antibodies of defined specificities. Proc Natl Acad Sci U S A. 1996;93(15):7843-7848.

57. Allan SE, et al. Generation of potent and stable human $\mathrm{CD} 4^{+} \mathrm{T}$ regulatory cells by activationindependent expression of FOXP3. Mol Ther. 2008;16(1):194-202.

58. Himmel ME, MacDonald KG, Garcia RV, Steiner TS, Levings MK. Helios ${ }^{+}$and Helios ${ }^{-}$cells coexist within the natural $\mathrm{FOXP}^{+} \mathrm{T}$ regulatory cell subset in humans. J Immunol. 2013;190(5):2001-2008.

59. Ye Q, et al. Engineered artificial antigen presenting cells facilitate direct and efficient expansion of tumor infiltrating lymphocytes. J Transl Med. 2011;9:131.

60. Jerome KR, Sloan DD, Aubert M. Measuring T-cell-mediated cytotoxicity using antibody to activated caspase 3. Nat Med. 2003;9(1):4-5.

61. Dijke IE, et al. Discarded human thymus is a novel source of stable long-lived therapeutic regulatory T cells. Am J Transplant. 2016;16(1):58-71.

62. Bacchetta R, Sartirana C, Levings MK, Bordignon C, Narula S, Roncarolo MG. Growth and expansion of human $\mathrm{T}$ regulatory type 1 cells are independent from TCR activation but require exogenous cytokines. Eur J Immunol. 2002;32(8):2237-2245.

63. Cooke KR, et al. An experimental model of idiopathic pneumonia syndrome after bone marrow transplantation: I. The roles of minor $\mathrm{H}$ antigen and endotoxin. Blood. 1996;88(8):3230-3239.

64. Hill GR, Crawford JM, Cooke KR, Brinson YS, Pan L, Ferrara JL. Total body irradiation and acute graft-versus-host disease: the role of gastrointestinal damage and inflammatory cytokines. Blood.1997;90(8):3204-3213. 